\title{
Molecular Self-Assembly: Quantifying the Balance between Intermolecular Attraction and Repulsion from Distance and Length Distributions
}

\author{
Christoph Schiel, Maximilian Vogtland, Ralf Bechstein, Angelika Kühnle,* and Philipp Maass*
}

Cite This: J. Phys. Chem. C 2020, 124, 21583-21590

Read Online

ACCESS | Llll Metrics \& More | 国 Article Recommendations | sl Supporting Information

ABSTRACT: Molecular self-assembly on surfaces constitutes a powerful method for creating tailor-made surface structures with dedicated functionalities. Varying the intermolecular interactions allows for tuning the resulting molecular structures in a rational fashion. So far, however, the discussion on the involved intermolecular interactions is often limited to attractive forces only. In real systems, the intermolecular interaction can be composed of both attractive and repulsive forces. Adjusting the balance between these interactions provides a promising strategy for extending the structural variety of molecular self-assembly on surfaces. However, this strategy relies on a method to quantify the involved interactions. Here, we investigate a molecular model system of 3-hydroxybenzoic acid (3-HBA)

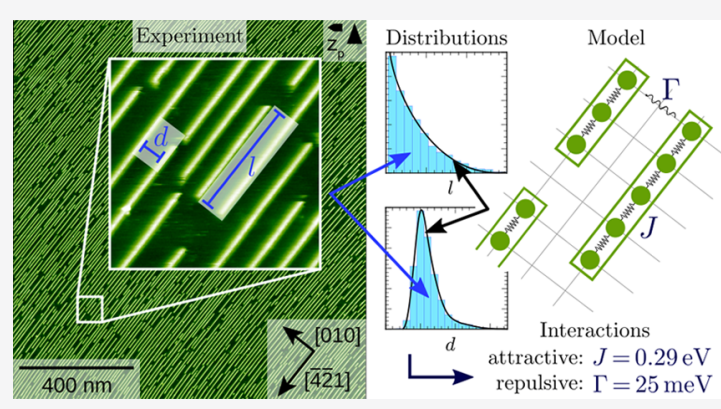
molecules on calcite (10.4) in a ultrahigh vacuum. This system offers both anisotropic short-range attraction and long-range repulsive dipolar interactions between molecules, resulting in the self-assembly of molecular stripes. We analyze the stripe-to-stripe distance distribution and the stripe length distribution and compare these distributions with analytical expressions from an anisotropic Ising model with additional repulsive interactions. We show that this approach allows the extraction of quantitative information about the strength of the attractive and repulsive interactions. Our work demonstrates how the detailed analysis of the self-assembled structures can be used to obtain quantitative insight into the molecule-molecule interactions.

\section{INTRODUCTION}

Molecular self-assembly has attracted great attention due to the impressive structural and functional variability that can be achieved with this versatile bottom-up method for supramolecular material synthesis. ${ }^{1}$ A clever design of the molecular building blocks allows controlling the resulting structures and tailoring them to the specific needs of a given application. ${ }^{2}$ The interaction of the molecules with the surface provides an additional way to tune the molecular structure formation. ${ }^{3-6}$

In the last few decades, the subtle balance between intermolecular and molecule-surface interactions has been explored to achieve an impressive multitude of various structures, ranging from perfectly ordered two-dimensional films ${ }^{7}$ over uni-directional rows ${ }^{8,9}$ to porous networks, ${ }^{10,11}$ and complex guest-host architectures. ${ }^{12-16}$ The vast majority of these studies have focused on attractive molecule-molecule interactions such as hydrogen bonding, van der Waals forces, $\pi-\pi$ interactions or electrostatic interactions. ${ }^{17}$ In contrast, repulsive molecule-molecule interactions have only rarely been studied for steering the structure formation., ${ }^{9-24}$ In the latter examples, the electrostatic repulsion between permanent as well as adsorption-induced electrical dipoles has been discussed as a promising way to enhance the structural complexity in molecular self-assembly on surfaces. Intermolecular repulsion leads to the formation of homogeneously dispersed individual molecules, ${ }^{18,19}$ extended rows with welldefined row-to-row distances, ${ }^{9,22}$ and islands ${ }^{24}$ and clusters ${ }^{25}$ with well-defined sizes.

However, so far, the interplay between attractive and repulsive interactions on the molecular structure formation has rarely been explored as a powerful strategy to control both the shape and the size of self-assembled molecular structures on surfaces. ${ }^{24,26,27}$ For systematically exploring the balance between molecular attraction and repulsion in molecular selfassembly, quantification of the involved interactions is essential.

Here, we present a molecular model system of adsorbed 3hydroxybenzoic acid molecules on a calcite (10.4) surface, see Figure 1, which provides both anisotropic attraction and repulsion. For this system, the molecular self-assembly has been shown to be governed by the balance between shortrange intermolecular attraction and long-range intermolecular repulsion. ${ }^{22,23}$ This balance results in the formation of

Received: July 21, 2020

Revised: September 1, 2020

Published: September 3, 2020 


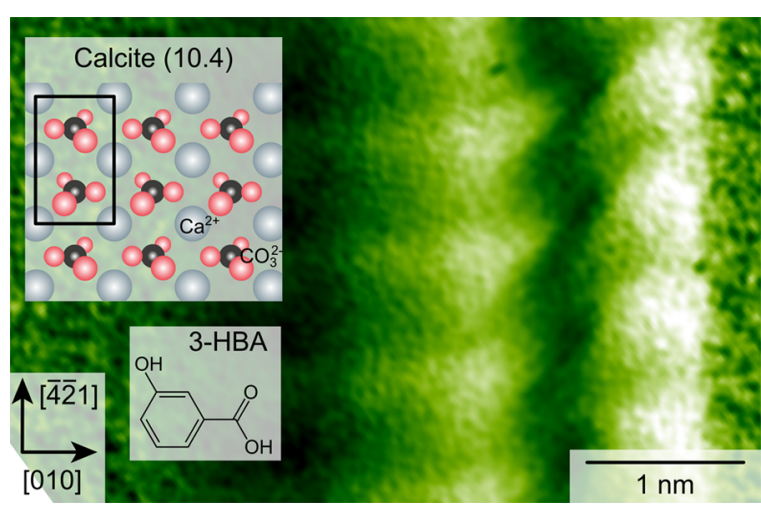

Figure 1. Model of the 3-hydroxybenzoic acid (3-HBA) molecule and the calcite (10.4) surface. The models are in scale to an underlying drift-corrected NC-AFM topography image of a single stripe.

molecular stripes with a coverage-dependent stripe-to-stripe distance distribution. $^{22}$

To determine the strength of the involved attractive and repulsive interactions, we consider an anisotropic Ising model with additional long-range dipole-dipole interactions. This model is generally applicable to stripe formation induced by intermolecular interactions. Based on a mean-field treatment we derive analytical expressions for stripe-to-stripe distance and stripe length distributions.

The theory is compared with the experimental data obtained from atomic force microscopy (AFM) images. An analysis of these images yields coverage-dependent stripe-to-stripe distance distributions as well as stripe length distributions. By fitting the theoretical predictions to the distance and length distributions we extract the strength of the attractive and repulsive molecule-molecule interactions. Our work provides an example of how the mesoscopic structural information can be used for gaining quantitative molecular-level insights into the driving forces at play.

\section{METHODS}

All dynamic atomic force microscopy (AFM) images shown in this work were acquired with a variable temperature atomic force microscope (VT-AFM XA from Scienta Omicron, Germany) operating under ultrahigh vacuum conditions $(p<$ $10^{-11}$ mbar). We used silicon cantilevers purchased from NanoWorld (Neuchâtel, Switzerland) with an eigen frequency of around $300 \mathrm{kHz}$ (type PPP-NCH) and a specified spring constant of $40 \mathrm{~N} / \mathrm{m}$. To remove contaminations and a possible oxide layer, the cantilevers were sputtered with $\mathrm{Ar}^{+}$at $2 \mathrm{keV}$ for $10 \mathrm{~min}$ prior to use.

The calcite crystals (Korth Kristalle GmbH, Germany) were prepared ex situ by mild ultrasonication in acetone and isopropanol for $15 \mathrm{~min}$ each. Inside the chamber, the crystals were degassed at about $580 \mathrm{~K}$ for $2 \mathrm{~h}$. After this degassing step, the crystals were cleaved and annealed at about $540 \mathrm{~K}$ for $1 \mathrm{~h}$. The quality of the crystal surface was then checked by collecting an image typically $100 \mathrm{~nm}^{2}$ in size.

The 3-hydroxybenzoic acid (3-HBA) molecules (99\% purity) were purchased from Sigma-Aldrich and used after degassing for $10 \mathrm{~min}$ at a temperature higher than $320 \mathrm{~K}$. A homebuilt Knudsen cell with a glass crucible was used for sublimation. For the crucible used here, a temperature of 309 $\mathrm{K}$ resulted in a flux of approximately 0.01 monolayers per minute $(\mathrm{ML} / \mathrm{min})$. During sublimation, the partial pressure in the chamber was in the range of $1 \times 10^{-12}$ mbar for 3-HBA $(m / z=137 \mathrm{u} / \mathrm{e})$ as measured with a mass spectrometer from MKS (e-Vision 2). For molecule deposition, the calcite sample was cooled to a temperature below $220 \mathrm{~K}$.

The AFM measurements were performed at a sample temperature of $290 \mathrm{~K}^{1}$ This temperature is chosen such that the dynamics are fast enough to ensure thermodynamic equilibrium but slow enough to minimize effects on the statistical analysis. The images were acquired with a pixel resolution of $4000 \times 4000 \mathrm{Px}$ and a speed of $0.32 \mathrm{~ms} / \mathrm{Px}$, resulting in a measurement time of roughly $3 \mathrm{~h}$ per image. The image size was $1500 \times 1500 \mathrm{~nm}^{2}$, yielding a resolution of 0.375 $\times 0.375 \mathrm{~nm}^{2} /$ Px.

We present measurement series for three different coverages, with multiple images measured at the same location. The number of images per coverage in each series differs since we had to sort out some of the images due to experimental difficulties. The remaining 14 images resulted in a total of 1758254 stripe-to-stripe distances $d$ and 17015 stripe lengths $l$.

To obtain the stripe-to-stripe distances and the length distributions from the AFM images we proceeded as follows. After a plane subtraction and line-by-line correction, ${ }^{28}$ the images were calibrated and corrected for linear drift. ${ }^{29}$ Each image was segmented using a trainable machine learning tool. ${ }^{30}$ Afterward, neighboring pixels were connected and the connected structures were fitted with a rectangle. ${ }^{31,32}$ All relevant data of the fit rectangles (centroid position, length $l$, and orientation) were collected and reconstructed as line
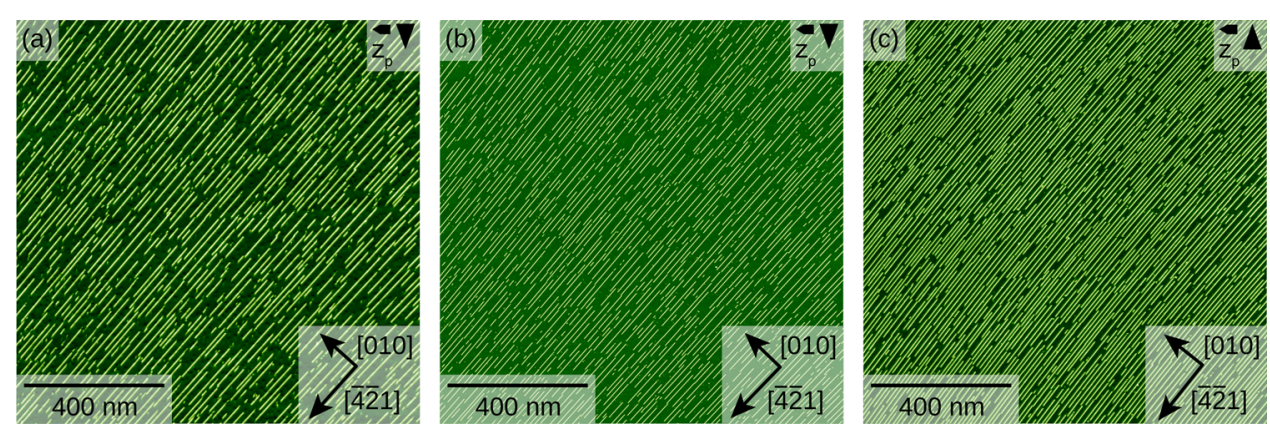

Figure 2. Representative atomic force microscopy (AFM) topography $\left(z_{\mathrm{p}}\right)$ images of 3-hydroxybenzoic acid (3-HBA) on calcite (10.4) from the three measured series I, II, and III at a temperature of $290 \mathrm{~K}$ and coverages (a) $\theta_{\mathrm{I}}=0.08 \mathrm{ML},(\mathrm{b}) \theta_{\mathrm{II}}=0.11 \mathrm{ML}$, and (c) $\theta_{\mathrm{III}}=0.16 \mathrm{ML}$. All images are cutouts with a size of $1150 \times 1150 \mathrm{~nm}^{2}$ and a resolution of $3446 \times 3446 \mathrm{Px}$. The fast (small arrow) and slow (large arrow) scan directions are given in the upper right corner. The surface directions are indicated by the arrows in the lower right corner. 
segments for further analysis using the SpatStat package within software $\mathrm{R}^{33,34}$ We sorted out stripes shorter than $5 \mathrm{~nm}$ since these are difficult to distinguish from wrongly fitted structures. For simplicity, we did not exclude stripes limited by image edges. We define the stripe-to-stripe distance as the distance between each 3-HBA dimer and its nearest-neighbor in the (010) direction. Thus, we obtained one distance per molecular dimer but only one length per stripe, which implies that the number of measured stripe distances is much larger than the number of stripe lengths.

\section{EXPERIMENTAL RESULTS}

When depositing 3-HBA molecules onto the (10.4) surface of calcite kept in a ultrahigh vacuum, the molecules self-assemble into double-rows, as reported previously. ${ }^{22}$ The molecular double rows can be identified in AFM images as stripes oriented along the $(4 \overline{2} 1)$ direction of the calcite crystal, see Figure 2. Two molecules, one out of each row, form the stripe basis with a periodicity of $0.8 \mathrm{~nm} .{ }^{22}$ We call this basis a $3-\mathrm{HBA}$ dimer. Each image in Figure 2 is a representative example of one of the three series, I-III, of measurements at a given coverage, where $\theta_{\mathrm{I}}=0.08 \mathrm{ML}$ (Figure 2a), $\theta_{\mathrm{II}}=0.11 \mathrm{ML}$ (Figure $2 \mathrm{~b}$ ), and $\theta_{\mathrm{III}}=0.16 \mathrm{ML}$ (Figure 2c). The coverage remains constant during the measurements for each series, i.e., desorption is not observed.

In Figure 3, we show the differences between stripe patterns in an example region of Figure $2 \mathrm{~b}$ and an image taken $6 \mathrm{~h}$

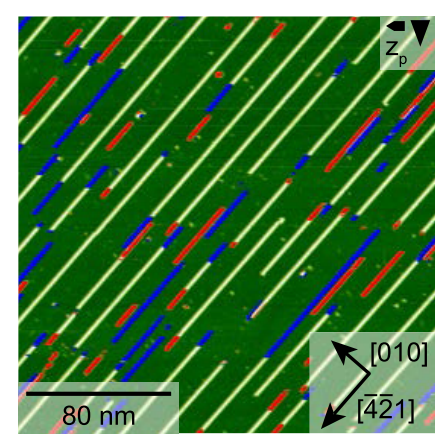

Figure 3. Comparison of a region of the image shown in Figure $2 b$ and an image taken $6 \mathrm{~h}$ before, demonstrating the redistribution of molecules. Areas where molecules disappear (appear) are marked in blue (red). before. Areas where the molecules disappear (appear) over time are marked in blue (red), i.e., the stripe pattern of the first image consists of the bright green and blue areas, while that of the second image consists of bright green and red areas. About $70 \%$ of the two stripe patterns overlap and the change of the blue area to the red area provides the evidence that the molecules are mobile at a sample temperature of $290 \mathrm{~K}$. Due to the strong overlap at a time separation of $6 \mathrm{~h}$, we expect that the statistics of stripe distances and lengths obtained for a single image is not strongly affected by the measurement time of roughly $3 \mathrm{~h}$ for one image.

From the last and the first image of series III with a time difference of $18 \mathrm{~h}$, we have generated the stripe-to-stripe distance distributions, as shown in Figure 4a, using a bin size of $0.5 \mathrm{~nm}$. A comparison of these two distributions reveals no significant difference. Both distributions exhibit a distinct maximum at a distance of $10-12 \mathrm{~nm}$, implying that the stripes are not randomly placed on the surface. A random placement would result in a geometric distribution. ${ }^{22}$ In addition, we have determined the stripe length distributions for the two images, which are shown in Figure $4 \mathrm{~b}$, for a bin size of $4 \mathrm{~nm}$. Again a comparison of the respective two length distributions shows no significant difference.

To conclude, during $18 \mathrm{~h}$ of measurement time appreciable rearrangements of the molecules occur but the stripe-to-stripe and the length distributions do not change. Hence, the stripe patterns can be regarded to reflect equilibrium structures. This justifies to analyze all images of each measurement series for improving the statistics.

Errors in our evaluation are dominated by fluctuations in the equilibrium structures, i.e., differences in counts obtained for different images in one series. In comparison, errors resulting from the image processing and further analysis are negligible. To provide information on the errors, we present histograms of each measurement series I-III in the Supporting Information, where we have marked the minimum and maximum number of counts in each of these histograms (found in each bin for different images belonging to each series).

In Figure 5a, we show the stripe-to-stripe distance obtained from all images in each series (four images for series I and II, and six images for series III). These distributions are coveragedependent, ${ }^{22}$ exhibiting a decrease of the mean distance $\left(\bar{d}_{\mathrm{I}}=\right.$ $24.1 \mathrm{~nm}, \bar{d}_{\mathrm{II}}=18.2 \mathrm{~nm}$, and $\left.\bar{d}_{\mathrm{III}}=12.2 \mathrm{~nm}\right)$, standard deviation $\left(\sigma_{\mathrm{I}}=12.2 \mathrm{~nm}, \sigma_{\mathrm{II}}=8.5 \mathrm{~nm}\right.$, and $\left.\sigma_{\mathrm{III}}=4.0 \mathrm{~nm}\right)$, and position of the maximum with increasing coverage.
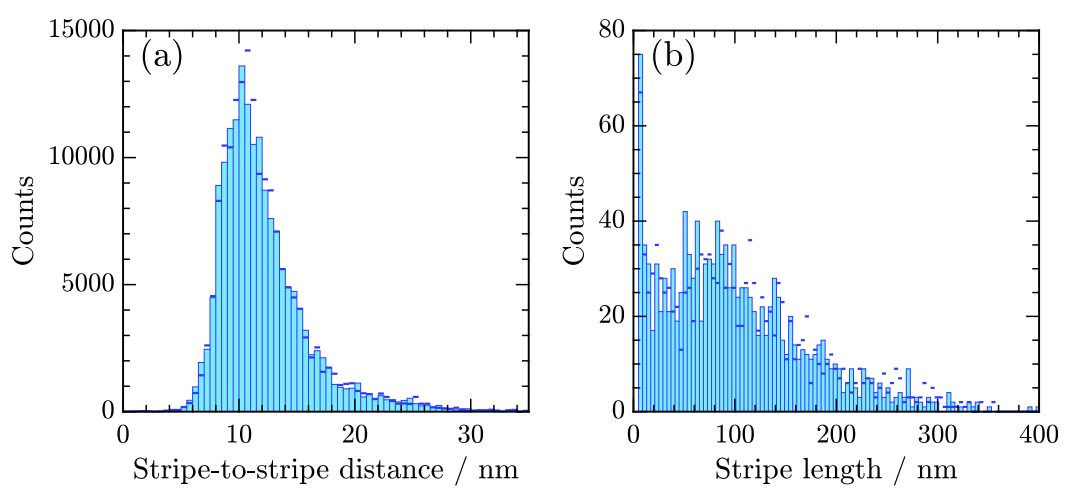

Figure 4. Comparison of histograms obtained from the first and last image of series III. The images have a time separation of $18 \mathrm{~h}$. In (a) the counts of stripe-to-stripe distances in bins $0.5 \mathrm{~nm}$ in size are shown, and in (b) the counts of stripe lengths in bins $4 \mathrm{~nm}$ in size. The bins give the histograms obtained from the first image, and the horizontal bars marked in blue indicate the corresponding counts from the last image. 

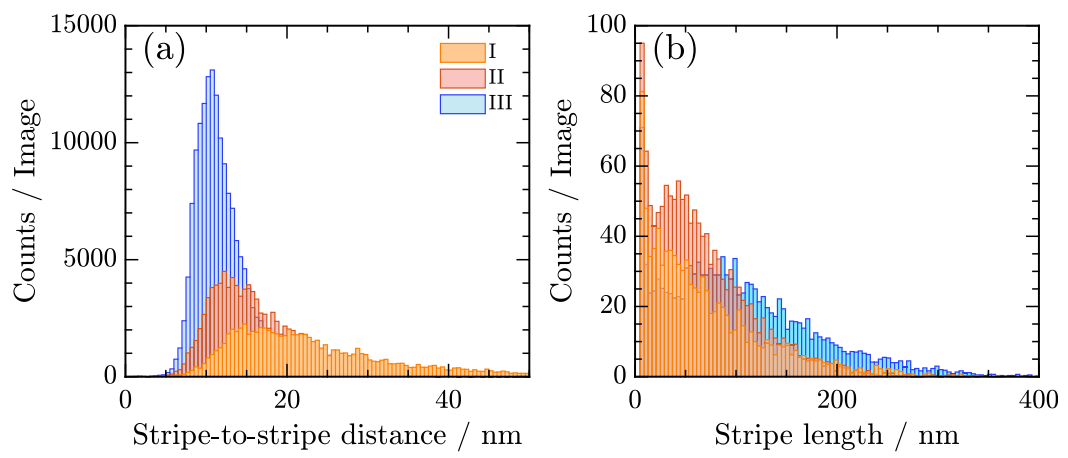

Figure 5. Histograms of (a) stripe-to-stripe distances and (b) stripe lengths obtained from all images in series I (0.08 ML, four images), II (0.11 ML, four images), and III (0.16 ML, six images) [color coding according to the legend in (a)]. The bin sizes are the same as those in Figure 4.

The corresponding length distributions are shown in Figure 5b. As explained above, the number of counts in each bin is much less than that for the stripe distances. Overall, the length distributions decrease monotonically for a large $l$. For the two higher coverages (series II and III), local maxima in the range $l$ $\approx 50-100 \mathrm{~nm}$ appear. A corresponding maximum, however, is not clearly detectable at the lowest coverage (series I).

The average total numbers of stripes per image are $N_{\mathrm{I}}=959$, $N_{\text {II }}=1370$, and $N_{\text {III }}=1284$ in series I-III. Fluctuations in the total stripe number per image are small with variations of less than $4 \%$. We determined mean lengths $\bar{l}\left(\bar{l}_{\mathrm{I}}=70.3 \mathrm{~nm}, \bar{l}_{\mathrm{II}}=\right.$ $65.8 \mathrm{~nm}$, and $\left.\bar{l}_{\text {III }}=104.4 \mathrm{~nm}\right)$ and respective standard deviations $\sigma_{l}\left(\sigma_{l, \mathrm{I}}=59.6 \mathrm{~nm}, \sigma_{l, \mathrm{II}}=49.0 \mathrm{~nm}\right.$, and $\sigma_{l, \mathrm{III}}=92.2$ $\mathrm{nm}$ ) for the three series. Although we can see and expect a global trend of increasing mean length and standard deviation with increasing coverage, both values are smaller for series II compared to series I.

\section{THEORETICAL MODELING}

For the equilibrated system of 3-HBA molecules on calcite, it has been proposed that repulsive interactions are caused by a charge transfer between the surface and molecules, leading to dipolar moments perpendicular to the surface. ${ }^{22}$ As the stripes are formed by dimers, it is convenient to consider these as molecular units occupying lattice sites. We refer to them as "particles". The lattice sites correspond to the anchoring positions on the calcite surface.

The analysis of AFM images shows that the stripes have a width of $2 \mathrm{~nm}$ and a periodicity of $0.8 \mathrm{~nm} .^{22}$ This can be represented by a rectangular lattice with spacings $a_{\|}=0.8 a_{0}$ in the stripe direction and $a_{\perp}=2 a_{0}$ perpendicular to it, where $a_{0}=$ $1 \mathrm{~nm}$ sets our length unit.

The interplay between attractive and dipolar interactions is described by the lattice gas Hamiltonian

$$
H=-\frac{J}{2} \sum_{i \mathrm{NN} j} n_{i} n_{j}+\frac{\Gamma}{2} \sum_{k, l} \frac{n_{k} n_{l}}{r_{k l}^{3}}
$$

where $n_{i}$ is the occupation number, i.e., $n_{i}=1$ if the site $i$ is occupied by a particle and zero otherwise. The sum over $i$ and $j$ is restricted to nearest-neighbor (NN) sites in the stripe direction corresponding to an anisotropic Ising model, and $r_{k l}$ is the (dimensionless) distance between sites $k$ and $l$. The interaction parameter $J>0$ quantifies the strength of the attractive nearest-neighbor interaction. The strength of the repulsive dipole interaction is given by

$$
\Gamma=\frac{p^{2}}{4 \pi \epsilon_{0} a_{0}^{3}}
$$

where $p$ is the dipole moment of one dimer and $\epsilon_{0}$ is the dielectric permeability of the vacuum.

In the following two subsections, we discuss analytical approaches to get insight into equilibrated stripe patterns for $\Gamma$ $=0$, and for $\Gamma>0$ based on approximate one-dimensional treatments. This allows one to determine the interaction parameters $J$ and $\Gamma$ by fitting analytical expressions to match experimentally observed stripe distances and length distributions. For convenient notation in the following theoretical treatment, the stripe length $l$ is given in units of $a_{\|}$and the stripe distance $d$ in units of $a_{\perp}$.

4.1. Stripe Formation for $\boldsymbol{\Gamma}=\mathbf{0}$. In the absence of dipolar interactions, the stripe positions in the perpendicular direction are uncorrelated. As a consequence, the stripe distance distribution $\Phi_{0}(d)$ is geometric, $\Phi_{0}(d)=\theta(1-\theta)^{d-1}$.

For deriving the stripe length distribution, we can focus on a one-dimensional row of stripes. A stripe of length $l$ corresponds to the occupation number sequence $01 \ldots 10$, i.e., a configuration of two zeros separated by $l$ ones. We denote the probability of such sequence by $q_{l}$. Knowing $q_{l}$ the stripe length distribution can be calculated as $\psi(l)=q_{l} / \sum_{l=1}^{\infty} q_{l}$.

To determine $q_{v}$, we introduce the conditional probabilities $w\left(n_{i+1} \mid n_{i}, n_{i-1}, \ldots, n_{1}\right)$ of finding occupation number $n_{i+1}$ if the occupation numbers $n_{i}, n_{i-1}, \ldots, n_{1}$ are given. In the grand canonical ensemble these satisfy the Markov property $w\left(n_{i+1} \mid n_{i}\right.$, $\left.n_{i-1}, \ldots, n_{1}\right)=w\left(n_{i+1} \mid n_{i}\right) .{ }^{35}$ Accordingly, $q_{l}=(1-\theta) w(1 \mid 0) w(1 \mid$ $1)^{l-1} w(0 \mid 1)$, where the factor $(1-\theta)$ accounts for the first zero in the sequence, and the product of $w(. I$.$) is the Markov chain$ corresponding to the occupation numbers in the sequence. The conditional probability $w(1 \mid 1)$ is given by $w(1 \mid 1)=$ $\chi_{2}(1,1) / \chi_{1}(1)$ with $\chi_{1}(1)=\theta$, and the joint probability $\chi_{2}(1,1)$ is equal to the equilibrium nearest-neighbor correlator ${ }^{36}$

$$
\begin{aligned}
C(J) & =\left\langle n_{i} n_{i+1}\right\rangle_{\mathrm{eq}} \\
& =\theta+\frac{1-\sqrt{1+4 \theta(1-\theta)\left(e^{J}-1\right)}}{2\left(e^{J}-1\right)}
\end{aligned}
$$

Hence, the $l$ dependence of $q_{l}$ is $\propto(C / \theta)^{l}$, and for the length distribution we obtain

$$
\Psi_{0}(l)=\frac{\theta-C(J)}{C(J)}\left(\frac{C(J)}{\theta}\right)^{l}
$$


in agreement with results earlier reported in ref 37. For $J \rightarrow 0$, $C(0)=\theta^{2}$ and we obtain the geometric distribution $\Psi_{0}(l)=(1$ $-\theta) \theta^{l-1}$.

4.2. Stripe Formation for $\boldsymbol{\Gamma}>0$. The dipolar interaction for $\Gamma>0$ leads to repulsion between pairs of particles belonging to the same stripe as well as to different stripes. This tends to shorten the stripes and increase the stripe distances. Compared to the case of $\Gamma=0$, the stripe distance distribution is more strongly affected than the length distribution because the latter is largely determined by the attractive nearestneighbor interaction $J$ (if $\Gamma<J$ ).

In fact, one can expect that the length distribution for large $l$ is still geometric as in eq 4 for $\Gamma=0$. This is because for each $\Gamma$ $>0$ there is a characteristic length scale of induced correlations by dipolar interactions. Considering long stripes to be composed of particle blocks on this length scale, the reasoning in the previous subsection leading to eq 4 is applicable with renormalized $C=C_{\text {eff }}(J, \Gamma)$ in eq 3 . Hence, the length distribution in the presence of dipolar interactions is expected to decay exponentially for large $l$ and to show deviations from the geometric shape at small stripe lengths.

Exact analytical solutions for the distance and length distributions are not available in the presence of competing attractive nearest-neighbor and dipolar interactions. We therefore rely on approximate treatments here.

As for the stripe lengths, it is instructive to first analyze whether a single isolated stripe can have an energetic minimum at a finite length. When increasing the length of this single stripe from $l$ to $l+1$, the energy changes by

$$
\Delta H(l)=-J+\Gamma \sum_{k=1}^{l} \frac{1}{k^{3}}
$$

For a minimum to occur, $\Delta H(l)$ must be negative for $l=1$ and positive for $l \rightarrow \infty$. This implies that $1<J / \Gamma<\zeta(3) \cong 1.202$, where $\zeta(3)$ is the Riemann zeta function (Apéry's constant). Accordingly, a finite single stripe can form only in a narrow regime of the interaction parameters $J$ and $\Gamma$. However, in a system consisting of many interacting stripes at a given coverage, the stripes can mutually stabilize each other at finite lengths for a wide range of $J$ and $\Gamma$.

Due to the fast convergence of the sum in eq 5, the energy change $\Delta H(l)$ for attaching one further particle to a stripe becomes essentially constant for $l \gtrsim 10$. Thus, we can expect eq 4 to hold for large $l$ with $C_{\text {eff }}(J, \Gamma)=C\left(J_{\text {eff }}\right)$, where

$$
J_{\text {eff }}=J-\zeta(3) \Gamma
$$

The corresponding approximate stripe length distribution is referred to as $\tilde{\Psi}(l)$.

We expect this distribution to have the same asymptotic behavior as the true length distribution $\Psi(l)$,i.e.,

$$
\Psi(l) \sim \tilde{\Psi}(l) \sim\left(\frac{C\left(J_{\text {eff }}\right)}{\theta}\right)^{l}, \quad l \rightarrow \infty
$$

Deviations from $\tilde{\Psi}(l)$ are expected to be significant for small $l$. If the effective nearest-neighbor interaction $J_{\mathrm{eff}}$ is attractive, i.e., $J>\zeta(3) \Gamma$, the energy change $\Delta H(l)$ in eq 5 is negative, implying that single particles or small stripes are energetically unfavorable compared to longer stripes. Accordingly, we expect $\Psi(l)$ to be smaller than $\tilde{\Psi}(l)$ for small $l$.

As for the stripe distance distribution $\Phi(d)$, we can assume that it is governed by the dipolar interaction between neighboring stripes in the perpendicular direction. Applying a mean-field approach similar to that introduced in ref 22 , we divide the two-dimensional stripe pattern into mutually independent one-dimensional parallel bands in the perpendicular direction. The bands are considered to have the same width $\bar{l}$, where $\bar{l}$ is the mean stripe lengths.

For each stripe appearing in a band, we consider it to span the whole band, i.e., to have length $l=\bar{l}$. In one band, the interaction $U(d)$ between two stripes at distance $d$ with dipole density $p / a_{\|}$is (integrating along both stripes with parametrization $s_{1}$ and $s_{2}$ )

$$
\begin{aligned}
& U(d)=\frac{p^{2}}{4 \pi \epsilon_{0} a_{\|}^{2}} \int_{-\bar{l} / 2}^{\bar{l} / 2} d s_{1} \int_{-\bar{l} / 2}^{\bar{l} / 2} d s_{2} \frac{1}{\left|x_{1}\left(s_{1}\right)-x_{2}\left(s_{2}\right)\right|^{3}} \\
& =\frac{p^{2}}{2 \pi \epsilon_{0} d a_{\|}^{2}}\left[\left(1+\frac{\bar{l}^{2}}{d^{2}}\right)^{1 / 2}-1\right]
\end{aligned}
$$

Hence, we have mapped each band onto a one-dimensional lattice occupied by particles with interaction $U(d)$ between neighboring stripes.

The mean occupation of lattice sites is fixed by the coverage $\theta$. In the presence of the purely repulsive $U(d)$, it can be viewed as resulting from a confinement pressure $f$ which hinders the particles to become infinitely separated and to give rise to a mean distance $\bar{d}$. Thus, our approximation $\tilde{\Phi}(d)$ of the stripe distance distribution is given by

$$
\tilde{\Phi}(d)=\frac{1}{Z} \exp (-\beta[f d+U(d)])
$$

where $Z=\sum_{d=1}^{\infty} \exp (-\beta[f d+U(d)])$ and $f$ is fixed by the condition

$$
\bar{d}=\sum_{d=1}^{\infty} d \tilde{\Phi}(d)
$$

\section{APPLICATION TO EXPERIMENTS}

The parameters $J$ and $\Gamma$ are estimated by fitting $\tilde{\Phi}(d)$ from eq 9aa to the distribution $\Phi(d)$, and by fitting eq 7 to the tail of $\Psi(l)$, where $\Phi(d)$ and $\Psi(l)$ are the distributions obtained in the experiments.

We first determine $\Gamma$, and hence $p=\sqrt{\Gamma 4 \pi \epsilon_{0} a_{0}^{3}}$, by fitting $\tilde{\Phi}(d)$ to $\Phi(d)$ with the experimental $\bar{l}$ in eq 8 . We then extract $J_{\text {eff }}$ by fitting the tail of $\Psi(l)$ which yields $J$ via eq 6 .

Figure 6 shows fitting of $\tilde{\Phi}(d)$ (circles, connected by solid lines) to $\Phi(d)$ (histogram) for each series, using the least square method. The optimal values of $\Gamma$ (and corresponding $p$ ) for each coverage are listed in Table 1 . In all three cases, the predicted curves match the experiment. The fitted dipole moment decreases from 7.0 to $5.8 \mathrm{D}$ with increasing $\theta$. When fixing the dipole moment to the mean $\bar{p}=6.3 \mathrm{D}$ of these values, the corresponding $\tilde{\Phi}(d)$ are also in good agreement with the experiment, as shown by the dashed black lines in Figure 6.

The mean $\bar{p}$ differs by about $1 \mathrm{D}$ from the optimal value for the smallest coverage. This raises the question on the sensitivity of the fitting with respect to $p$. We thus analyze how $\tilde{\Phi}(d)$ deviates from $\Phi(d)$ for even larger differences of $p$ from its optimal value. For values $p_{>}=8.3 \mathrm{D}$ and $p_{<}=4.3 \mathrm{D}$ larger and smaller by $2 \mathrm{D}, \tilde{\Phi}(d)$ is shown in the inset of Figure 

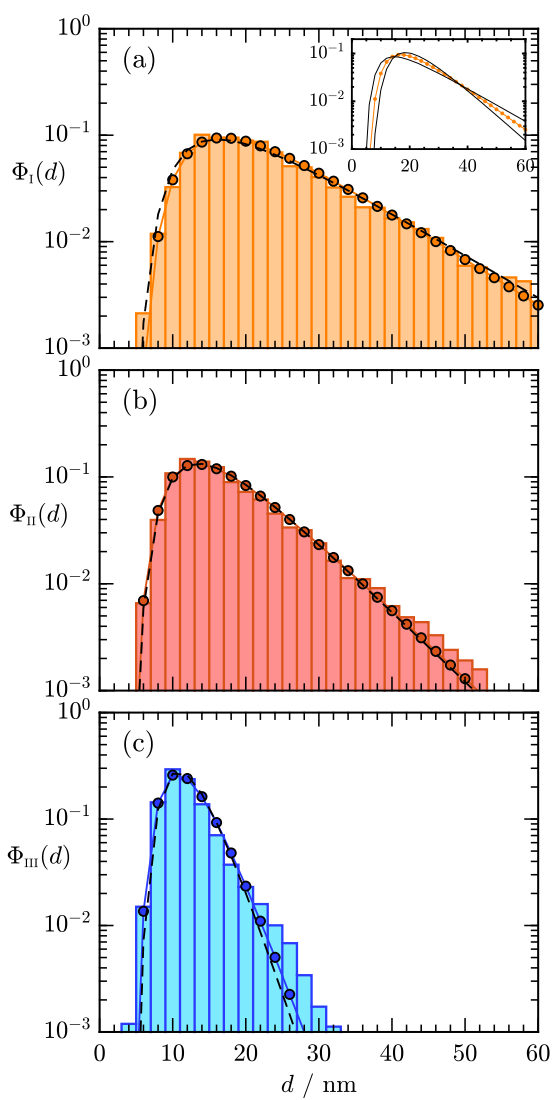

Figure 6. Histograms of the measured distance distributions $\Phi(d)$ for the three different coverages (a) $\theta_{\mathrm{I}}=0.08 \mathrm{ML}$, (b) $\theta_{\mathrm{II}}=0.11 \mathrm{ML}$, and (c) $\theta_{\text {III }}=0.16 \mathrm{ML}$ in comparison with the fitted theoretical distributions $\tilde{\Phi}(d)$ (circles, connected by solid lines of the corresponding color). Dashed black lines correspond to $\tilde{\Phi}(d)$ with the mean dipole moment of $\bar{p}=6.3 \mathrm{D}$. The inset in (a) shows the fitted $\tilde{\Phi}(d)$ for $\theta_{\mathrm{I}}$ (circles, connected by orange line) compared to $\tilde{\Phi}(d)$ for $p_{>}=8.3 \mathrm{D}$ and $p_{<}=4.3 \mathrm{D}$ (black lines).

Table 1. Parameters Obtained by Fitting the Theoretical Model to the Experimental Stripe Distance and Length Distributions for the Three Different Coverages (Series IIII)

\begin{tabular}{ccccc} 
series & $\theta / \mathrm{ML}$ & $p / \mathrm{D}$ & $\Gamma / \mathrm{meV}$ & $\mathrm{J} / \mathrm{eV}$ \\
I & 0.08 & $7.0 \pm 1$ & $31 \pm 8$ & $0.32 \pm 0.04$ \\
II & 0.11 & $6.1 \pm 1$ & $24 \pm 8$ & $0.28 \pm 0.04$ \\
III & 0.16 & $5.8 \pm 1$ & $21 \pm 8$ & $0.28 \pm 0.04$ \\
\hline
\end{tabular}

6a. As can be seen from this inset, deviations to $\tilde{\Phi}(d)$ for the optimal $p$ value are now clearly visible. We thus conclude that the error in our estimate is about $\pm 1 \mathrm{D}$.

Taking $\bar{p}$ as the dipole moment of the 3-HBA dimer yields a dipole moment $p / 2=3.2 \mathrm{D}$ for the single molecule, in fair agreement with our former estimate. ${ }^{22}$

Having determined $\Gamma$, we now analyze the stripe length distribution to determine J. Figure 7 shows the measured length distributions $\Psi(l)$ for each series (circles). The distributions are determined using bins of varying sizes with approximately equal amount of events in each bin. As expected, all distributions show an exponential decay for large $l$. Deviations for smaller lengths $l \lesssim 100 \mathrm{~nm}$ occur because of the reasons discussed in Section 4.2. The solid lines are fitted

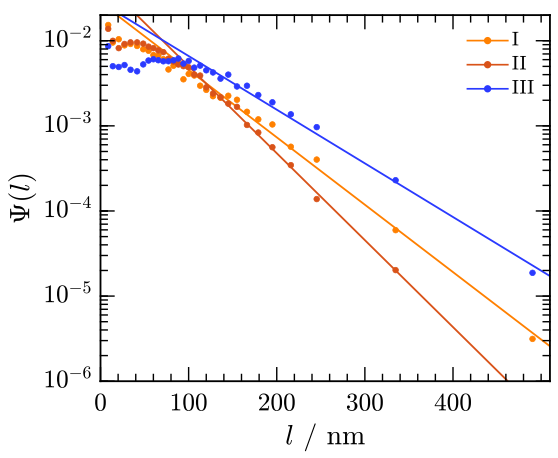

Figure 7. Stripe length distributions $\Psi(l)$ for the three different coverages (I-III, circles) with fits to the exponential tails for $l>100$ $\mathrm{nm}$ (solid lines).

to exponential decays for $l>100 \mathrm{~nm}$. According to eq 7, the decay length of $\Psi(l) \sim e^{-l / l_{0}}$ is

$$
l_{0}=\frac{a_{\|}}{\ln \left(C\left(J_{\text {eff }}\right) / \theta\right)}
$$

The characteristic decay length $l_{0}$ for each experimental distribution thus yields a value $J_{\text {eff }}$ via eq 10 in combination with eq 3. The interaction parameters $J$ then follow from eq 6 and are listed in the fifth column of Table 1 . These values lie at around $0.29 \mathrm{eV}$. Our final estimate of the analysis is $p=6.3 \pm 1$ $\mathrm{D}$ and $J=0.29 \pm 0.04 \mathrm{eV}$.

The interaction strength $J$ is in the range of hydrogen bonds and lower than $\approx 0.7 \mathrm{eV}$ between two molecules in a carboxylic acid dimer. $^{38-40}$

\section{DISCUSSION AND CONCLUSIONS}

In summary, we have presented an approach to estimate the strengths of short-range attractive and long-range repulsive interactions between 3-HBA molecules on a calcite surface by the analysis of stripe-to-stripe distance distributions $\Phi(d)$ and stripe length distributions $\Psi(l)$.

Experimental distributions were determined from the analysis of three AFM image series with different coverages $0.08,0.11$, and $0.16 \mathrm{ML}$ at a temperature of $290 \mathrm{~K}$. The measurements of theses series spanned time intervals of up to $18 \mathrm{~h}$. A comparison between distributions of individual images in the same series strongly suggests that the stripe patterns are in a thermodynamic equilibrium.

The attractive interaction responsible for the stripe formation was considered to be an effective one with strength $J$ between neighboring 3-HBA dimers, without resorting to details of the molecular structure. The long-range repulsive interaction is modeled as dipole-dipole interaction of characteristic strength $\Gamma$ as previously proposed in ref 22 . It is believed to be caused by charge transfer between the surface and 3-HBA molecules. As these molecules have specific anchoring sites on the calcite surface, the system could be described by a lattice gas model corresponding to an anisotropic Ising model with additional dipolar interactions.

Based on this model, we developed mean-field approaches to derive approximate expressions for the stripe distance and length distributions with $J$ and $\Gamma$ as parameters. Fitting these parameters to the experimental distributions we obtained the estimates $J=0.29 \pm 0.04 \mathrm{eV}$ and $p=6.3 \pm 1 \mathrm{D}$ for the dipole moment $p \propto \sqrt{ } \Gamma$ of a 3-HBA dimer. 
The modeling approach presented here is applicable also to other molecular systems self-assembling into stripe patterns, if the stripe formation is dominated by short-range attractive molecule-molecule interactions. In general, one can expect additional long-range electrostatic interactions to be present. Their impact on the structure formation depends on their type (e.g., dipolar, quadrupolar) and strength, but the core of our methodology is independent of these features.

The mean-field treatment, however, requires the formation of structures with long stripes arranged into patterns with large overlaps between neighboring parallel stripes. This requirement is fulfilled only if the repulsive interaction is not too strong in comparison to the attractive one, and if the coverage is not too small. The coverage must not be too high either because otherwise the structure will no longer be composed of individual stripes. For determining the respective limits of our mean-field treatment, extensive simulations of the many-body problem are needed, which is left for future research.

As long as the aforementioned requirements are met, other types of interactions can be accounted for by minor adjustments of the mean-field approach. As for the stripe distance distribution, only the effective interaction potential $U(d)$ between stripes in eq 8 needs to be modified. As for the stripe length distribution, we expect a length scale to exist beyond which correlations within a stripe can be renormalized to an effective nearest-neighbor interaction between segments. The interplay between attractive and repulsive interaction in $\Phi(l)$ can then be accounted for by one effective coupling parameter analogous to $J_{\text {eff }}$ in eq 6 .

From a general point of view, it should be scrutinized whether modeling with static dipole moment is appropriate. Our use of a static dipole moment here relies on the assumption of an approximately fixed amount of charges transferred between the surface and each molecule. The results in Table 1 indicate a decreasing dipole moment with increasing coverage. This can be interpreted by a dynamic dipole moment which becomes smaller to compensate for additional repulsive interactions with increasing number of molecules. A change of the molecule-surface interaction as a response to a repulsive interaction has been reported earlier in refs 41-43.

Dynamical dipole moments can be coped within a theoretical treatment by introducing molecular polarizability for the molecules. This leads to varying dipole moments in dependence of their local environment. How important these variations are, is presently unknown. The uncertainties of the values in Table 1 and the rather narrow coverage range 0.08$0.16 \mathrm{ML}$ does not allow us to give a firm assessment on how strong the effects of a dynamical dipole moment are. Additional investigations with a wider range of coverages are needed. Further experimental and theoretical research in this direction will offer promising perspectives to gain deeper insight into the impact of the interplay between repulsive and attractive interactions on molecular self-assembly.

\section{ASSOCIATED CONTENT}

\section{(s) Supporting Information}

The Supporting Information is available free of charge at https://pubs.acs.org/doi/10.1021/acs.jpcc.0c06676.

Histograms of stripe-to-stripe distances and stripe lengths for each of the measurement series I-III with errors quantified by equilibrium image-to-image fluctuations (PDF)

\section{AUTHOR INFORMATION}

\section{Corresponding Authors}

Angelika Kühnle - Fakultät für Chemie, Universität Bielefeld, D-33615 Bielefeld, Germany; ๑ orcid.org/0000-0001-54974685; Email: kuehnle@uni-bielefeld.de

Philipp Maass - Fachbereich Physik, Universität Osnabrück, D49076 Osnabrück, Germany; Email: maass@uniosnabrueck.de

\section{Authors}

Christoph Schiel - Fachbereich Physik, Universität Osnabrück, D-49076 Osnabrück, Germany

Maximilian Vogtland - Fakultät für Chemie, Universität Bielefeld, D-33615 Bielefeld, Germany

Ralf Bechstein - Fakultät für Chemie, Universität Bielefeld, D33615 Bielefeld, Germany

Complete contact information is available at:

https://pubs.acs.org/10.1021/acs.jpcc.0c06676

\section{Notes}

The authors declare no competing financial interest.

\section{ACKNOWLEDGMENTS}

We thank the German Research Foundation (DFG) for funding (KU 1980/10-1) "Intermolecular Repulsion in Molecular Self-Assembly on Bulk Insulator Surfaces".

\section{ADDITIONAL NOTE}

${ }^{I}$ In the AFM, the temperature is read out at the sample stage using a Pt100 sensor $3 \mathrm{~cm}$ away from the sample. According to the manufacturer, the temperature difference between the sample and the sample readout position in the AFM is smaller than $10 \mathrm{~K}$.

\section{REFERENCES}

(1) Whitesides, G. M.; Mathias, J. P.; Seto, C. T. Molecular selfassembly and nanochemistry: a chemical strategy for the synthesis of nanostructures. Science 1991, 254, 1312-1319.

(2) Lehn, J. M. Supramolecular Chemistry - Scope and Perspectives Molecules, Supermolecules, and Molecular Devices (Nobel lecture). Angew. Chem., Int. Ed. 1988, 27, 89-112.

(3) Barth, J. V.; Costantini, G.; Kern, K. Engineering atomic and molecular nanostructures at surfaces. Nature 2005, 437, 671-679.

(4) Auwärter, W.; Schiffrin, A.; Weber-Bargioni, A.; Pennec, Y.; Riemann, A.; Barth, J. V. Molecular nanoscience and engineering on surfaces. Int. J. Nanotechnol. 2008, 5, 1171-1193.

(5) Mali, K. S.; Pearce, N.; De Feyter, S.; Champness, N. R. Frontiers of supramolecular chemistry at solid surfaces. Chem. Soc. Rev. 2017, 46, 2520-2542.

(6) Otero, R.; Gallego, J. M.; de Parga, A. L.; Martín, N.; Miranda, R. Molecular self-assembly at solid surfaces. Adv. Mater. 2011, 23, 51485176.

(7) Miwa, J. A.; Cicoira, F.; Bedwani, S.; Lipton-Duffin, J.; Perepichka, D. F.; Rochefort, A.; Rosei, F. Self-assembly of rubrene on copper surfaces. J. Phys. Chem. C 2008, 112, 10214-10221.

(8) Kühnle, A.; Molina, L. M.; Linderoth, T. R.; Hammer, B.; Besenbacher, F. Growth of Unidirectional Molecular Rows of Cysteine on $\mathrm{Au}(110)-(1 \times 2)$ Driven by Adsorbate-Induced Surface Rearrangements. Phys. Rev. Lett. 2004, 93, No. 086101.

(9) Schiffrin, A.; Riemann, A.; Auwärter, W.; Pennec, Y.; WeberBargioni, A.; Cvetko, D.; Cossaro, A.; Morgante, A.; Barth, J. V. Zwitterionic self-assembly of L-methionine nanogratings on the $\mathrm{Ag}(111)$ surface. Proc. Natl. Acad. Sci. U.S.A. 2007, 104, 5279-5284.

(10) Kepčija, N.; Zhang, Y. Q.; Kleinschrodt, M.; Björk, J.; Klyatskaya, S.; Klappenberger, F.; Ruben, M.; Barth, J. V. Steering 
On-Surface Self-Assembly of High-Quality Hydrocarbon Networks with Terminal Alkynes. J. Phys. Chem. C 2013, 117, 3987-3995.

(11) Pawin, G.; Wong, K. L.; Kwon, K. Y.; Bartels, L. A homomolecular porous network at a $\mathrm{Cu}(111)$. Surf. Sci. 2006, 313, 961-962.

(12) Stepanow, S.; Lingenfelder, M.; Dmitriev, A.; Spillmann, H.; Delvigne, E.; Lin, N.; Deng, X.; Cai, C.; Barth, J. V.; Kern, K. Steering molecular organization and host-guest interactions using twodimensional nanoporous coordination systems. Nat. Mater. 2004, 3, 229-233.

(13) Theobald, J. A.; Oxtoby, N. S.; Phillips, M. A.; Champness, N. R.; Beton, P. H. Controlling molecular deposition and layer structure with supramolecular surface assemblies. Nature 2003, 424, 10291031.

(14) Klyatskaya, S.; Klappenberger, F.; Schlickum, U.; Kühne, D.; Marschall, M.; Reichert, J.; Decker, R.; Krenner, W.; Zoppellaro, G.; Brune, H.; et al. Surface-Confined Self-Assembly of Di-carbonitrile Polyphenyls. Adv. Funct. Mater. 2011, 21, 1230-1240.

(15) Écija, D.; Seufert, K.; Heim, D.; Auwärter, W.; Aurisicchio, C.; Fabbro, C.; Bonifazi, D.; Barth, J. V. Hierarchic Self-Assembly of Nanoporous Chiral Networks with Conformationally Flexible Porphyrins. ACS Nano 2010, 4, 4936-4942.

(16) Kühne, D.; Klappenberger, F.; Decker, R.; Schlickum, U.; Brune, H.; Klyatskaya, S.; Ruben, M.; Barth, J. V. Self-Assembly of Nanoporous Chiral Networks with Varying Symmetry from Sexiphenyl-dicarbonitrile on $\operatorname{Ag}(111)$. J. Phys. Chem. C 2009, 113, 17851-17859.

(17) Barth, J. V. Molecular architectonic on metal surfaces. Annu. Rev. Phys. Chem. 2007, 58, 375-407.

(18) Yokoyama, T.; Takahashi, T.; Shinozaki, K.; Okamoto, M. Quantitative Analysis of Long-Range Interactions between Adsorbed Dipolar Molecules on Cu(111). Phys. Rev. Lett. 2007, 98, No. 206102.

(19) Fernández-Torrente, I.; Monturet, S.; Franke, K. J.; Fraxedas, J.; Lorente, N.; Pascual, J. I. Long-range repulsive interaction between molecules on a metal surface induced by charge transfer. Phys. Rev. Lett. 2007, 99, No. 176103.

(20) Tomba, G.; Stengel, M.; Schneider, W. D.; Baldereschi, A.; De Vita, A. Supramolecular Self-Assembly Driven by Electrostatic Repulsion: The 1D Aggregation of Rubrene Pentagons on $\mathrm{Au}(111)$. ACS Nano 2010, 4, 7545-7551.

(21) Wen, X. J.; Lin, Y. X.; Huang, Z. C.; Diao, M. X.; Zhao, W. H.; Dai, J. X.; Xing, L. B.; Zhu, H.; Peng, Z. T.; Liu, D.; et al. Long-Range Ordered Structures of Corannulene Governed by Electrostatic Repulsion and Surface-State Mediation. J. Phys. Chem. Lett. 2019, $10,6800-6806$.

(22) Neff, J. L.; Söngen, H.; Bechstein, R.; Maass, P.; Kühnle, A. Long-range order induced by intrinsic repulsion on an insulating substrate. J. Phys. Chem. C 2015, 119, 24927-24931.

(23) Neff, J. L.; Richter, A.; Söngen, H.; Venturini, C.; Gourdon, A.; Bechstein, R.; Kühnle, A. Generic nature of long-range repulsion mechanism on a bulk insulator? Faraday Discuss. 2017, 204, 419-428.

(24) Della Pia, A.; Riello, M.; Floris, A.; Stassen, D.; Jones, T. S.; Bonifazi, D.; De Vita, A.; Costantini, G. Anomalous Coarsening Driven by Reversible Charge Transfer at Metal-Organic Interfaces. ACS Nano 2014, 8, 12356-12364.

(25) Murphy, C. J.; Miller, D. P.; Simpson, S.; Baggett, A.; Pronschinske, A.; Liriano, M. L.; Therrien, A. J.; Enders, A.; Liu, S.-Y.; Zurek, E.; et al. Charge-Transfer-Induced Magic Cluster Formation of Azaborine Heterocycles on Noble Metal Surfaces. J. Phys. Chem. C 2016, 120, 6020-6030.

(26) Henneke, C.; Felter, J.; Schwarz, D.; Stefan Tautz, F.; Kumpf, C. Controlling the growth of multiple ordered heteromolecular phases by utilizing intermolecular repulsion. Nat. Mater. 2017, 16, 628-633.

(27) Teichert, C. Opposite interaction matters. Nat. Mater. 2017, 16, 604-606.

(28) Neučas, D.; Klapetek, P. Gwyddion: an open-source software for SPM data analysis. Open Phys. 2012, 10, 181-188.

(29) Rahe, P.; Bechstein, R.; Kühnle, A. Vertical and lateral drift corrections of scanning probe microscopy images. J. Vac. Sci. Technol.,
B: Nanotechnol. Microelectron.: Mater., Process., Meas., Phenom. 2010, 28, C4E31-C4E38.

(30) Arganda-Carreras, I.; Kaynig, V.; Rueden, C.; Eliceiri, K. W.; Schindelin, J.; Cardona, A.; Seung, H. S. Trainable Weka Segmentation: a machine learning tool for microscopy pixel classification. Bioinformatics 2017, 33, 2424-2426.

(31) Legland, D.; Arganda-Carreras, I.; Andrey, P. MorphoLibJ: integrated library and plugins for mathematical morphology with ImageJ. Bioinformatics 2016, 32, 3532-3534.

(32) Schindelin, J.; Arganda-Carreras, I.; Frise, E.; Kaynig, V.; Longair, M.; Pietzsch, T.; Preibisch, S.; Rueden, C.; Saalfeld, S.; Schmid, B.; et al. Fiji: an open-source platform for biological-image analysis. Nat. Methods 2012, 9, 676-682.

(33) R Core Team, R: A Language and Environment for Statistical Computing. R Foundation for Statistical Computing: Vienna,Austria, http://www.R-project.org/ 2020.

(34) Baddeley, A.; Rubak, E.; Turner, R. Spatial Point Patterns: Methodology and Applications with R, Chapman \& Hall Book; CRC Press: Boca Raton and London and New York, 2016.

(35) Buschle, J.; Maass, P.; Dieterich, W. Exact density functionals in one dimension. J. Phys. A: Math. Gen. 2000, 33, L41.

(36) Dierl, M.; Einax, M.; Maass, P. One-dimensional transport of interacting particles: Currents, density profiles, phase diagrams, and symmetries. Phys. Rev. E 2013, 87, No. 062126.

(37) Yilmaz, M.; Zimmermann, F. M. Exact cluster size distribution in the one-dimensional Ising model. Phys. Rev. E 2005, 71, No. 026127.

(38) Steiner, T. The Hydrogen Bond in the Solid State. Angew. Chem., Int. Ed. 2002, 41, 48-76.

(39) Martsinovich, N.; Troisi, A. Modeling the Self-Assembly of Benzenedicarboxylic Acids Using Monte Carlo and Molecular Dynamics Simulations. J. Phys. Chem. C 2010, 114, 4376-4388.

(40) Tzeli, D.; Petsalakis, I. D.; Theodorakopoulos, G.; Ajami, D.; Rebek, J. Theoretical study of free and encapsulated carboxylic acid and amide dimers. Int. J. Quantum Chem. 2013, 113, 734-739.

(41) Della Pia, A.; Riello, M.; Floris, A.; Stassen, D.; Jones, T. S.; Bonifazi, D.; de Vita, A.; Costantini, G. Anomalous coarsening driven by reversible charge transfer at metal-organic interfaces. ACS Nano 2014, 8, 12356-12364.

(42) Duhm, S.; Bürker, C.; Niederhausen, J.; Salzmann, I.; Hosokai, T.; Duvernay, J.; Kera, S.; Schreiber, F.; Koch, N.; Ueno, N.; et al. Pentacene on $\operatorname{Ag}(111)$ : correlation of bonding distance with intermolecular interaction and order. ACS Appl. Mater. Interfaces 2013, 5, 9377-9381.

(43) Fraxedas, J.; García-Gil, S.; Monturet, S.; Lorente, N.; Fernández-Torrente, I.; Franke, K. J.; Pascual, J. I.; Vollmer, A.; Blum, R.-P.; Koch, N.; et al. Modulation of Surface Charge Transfer through Competing Long-Range Repulsive versus Short-Range Attractive Interactions. J. Phys. Chem. C 2011, 115, 18640-18648. 